\title{
Perturbative Reheating and Gravitino Production in Inflationary Models
}

\author{
Raghavan Rangarajan* \\ Theoretical Physics Division, Physical Research Laboratory, Navrangpura, Ahmedabad 380 009, India \\ Narendra Sahu团 \\ Department of Physics, Lancaster University, Lancaster, LA1 4YB, UK
}

\begin{abstract}
The low reheat temperature at the end of inflation from the gravitino bound constrains the creation of heavy Majorana neutrinos associated with models of leptogenesis. However, a detailed view of the reheating of the Universe at the end of inflation implies that the maximum temperature during reheating, $T_{\max }$, can be orders of magnitude higher than the final reheat temperature. This then allows for the production of the heavy Majorana neutrinos needed for leptogenesis. We carry out the complementary calculation of the gravitino production during reheating and its dependence on $T_{\max }$. We find that the gravitino abundance generated during reheating for a quartic potential is comparable to the standard estimate of the abundance generated after reheating and study its consequences for leptogenesis.
\end{abstract}

Keywords: Inflationary cosmology, reheating, gravitino abundance

PACS numbers: 98.80.-k,98.80.Cq

\section{INTRODUCTION}

It is presumed that the early universe went through a period of inflation and then reheated to create the radiation dominated epoch. If nature is supersymmetric then during the process of reheating many supersymmetric particles would have been produced, which can have important cosmological consequences. In particular, the production of gravitinos in the early universe and their subsequent evolution including decays has attracted attention. Stable gravitinos can overclose the universe while unstable gravitinos can affect the expansion rate of the universe during eras prior to their decay. The decay products of unstable gravitinos can also overclose the universe or affect light element abundances generated during nucleosynthesis. These cosmological consequences are a function of the gravitino energy density, $\rho_{\tilde{G}}=m_{\tilde{G}} n_{\tilde{G}}$, where $m_{\tilde{G}}$ and $n_{\tilde{G}}$ are the mass and number density of gravitinos. In an inflationary universe, $n_{\tilde{G}}$ is a function of the reheat temperature. Therefore, for a fixed $m_{\tilde{G}}$, often taken to be $O(100 \mathrm{GeV}-1 \mathrm{TeV})$, cosmological constraints on the energy density of gravitinos provide an upper bound on the reheat temperature [1, 2, 3, 4, 5, 6, 67, 8, 9, 10, 11].

The number density of gravitinos is usually obtained by considering gravitino production in the radiation dominated era following reheating, as in Refs. [1, 2, 3, 4, 4, 5, 6, 7, 8, 9, 10, 11], and it is presumed that $n_{\tilde{G}}=0$ at the beginning of the radiation dominated era. Gravitinos are produced through thermal scattering and the final gravitino abundance is found to be proportional to the reheat temperature, $T_{\text {reh }} . T_{\text {reh }}$ is the temperature of the thermal plasma at the beginning of the radiation dominated era at $t_{\text {reh }}$ when the inflaton field has decayed and the energy density of the universe is dominated by the inflaton decay products. The cosmological constraints on $n_{\tilde{G}}$ then provide an upper bound on $T_{\text {reh }} \lesssim 10^{6-9} \mathrm{GeV}$.

Such an analysis is consistent with the instantaneous decay approximation in which one assumes that reheating is instantaneous and therefore $T_{\text {reh }}$ is the maximum temperature during reheating. However, a more detailed understanding of (perturbative) reheating indicates that during reheating the temperature initially rises to a maximum temperature $T_{\max }$ and then falls to $T_{\text {reh }}$ 12, 13. In fact the maximum temperature during the course of reheating can be as high as $10^{3} T_{\text {reh }} 14$.

Earlier works have considered whether sufficient number densities of heavy GUT gauge and Higgs bosons, or right-handed Majorana neutrinos, required for GUT baryogenesis or leptogenesis respectively, can be generated with a high $T_{\max }$. They find that leptogenesis with Majorana neutrinos of mass $\sim 10^{10} \mathrm{GeV}$ is feasible. However it is then legitimate to ask if harmful gravitinos are also produced during the course of reheating. A priori one might expect large production with a $T_{\max }$ dependent abundance. This could have serious implications for leptogenesis scenarios that invoke large $T_{\max }[13,14,15]$.

In Ref. 16 we considered this issue for an inflaton with a potential of the form $V=\frac{1}{2} m^{2} \phi^{2}$ during reheating. We find that the abundance of gravitinos produced during reheating is $1 / 3$ of that produced in the radiation dominated epoch. Our estimate for the final gravitino abundance is of the same order as that obtained in Ref. [17] using a numerical analysis (also for a quadratic potential). Gravitino production including the reheating era contribution has also been obtained numerically in Refs. [18, 19]. In this article we investigate the gravitino abundance generated during the course of reheating in 
inflationary models with a potential $V=(\lambda / 4) \phi^{4}$ during reheating. The difference between the two scenarios lies in the different equations of state for an oscillating scalar field with a $\phi^{2}$ and a $\phi^{4}$ potential. In the two scenarios the oscillating scalar field behaves like non-relativistic and relativistic matter respectively, i.e., $\rho_{\phi} \propto R^{-3}, R^{-4}$ respectively [20]. This affects the Hubble expansion rate in the Boltzmann equation for radiation and gravitinos during reheating, and the source term for radiation during reheating, and thus the abundance of gravitinos produced by the scattering of the thermalised radiation.

For the $\phi^{4}$ potential we find that the gravitino abundance generated during reheating is about $49 \%$ of the gravitinos produced in the subsequent radiation dominated epoch. The gravitino abundance generated during reheating is a function of $T_{\max }$ but, as in the quadratic potential case, the abundance can be re-expressed as a function of $T_{\text {reh }}$ only. Including the contribution from the reheating era and then applying the cosmological constraints on the total gravitino abundance lowers the upper bound on $T_{\text {reh }}$ by a factor of $3 / 2$. This does not have a serious impact on leptogenesis scenarios discussed above.

Our results in Ref. [16] and in this article are valid for chaotic inflation models and for models of inflation where one can approximate the inflaton potential during reheating by a $\phi^{2}$ or $\phi^{4}$ term. However they are not valid for a reheating scenario that includes preheating 21,22 , 23, 24]. ${ }^{1}$ Gravitino production during preheating has been considered in Ref. 18, 28, 29, 30, 31, 32, 33, 34, 35.

\section{PERTURBATIVE REHEATING IN INFLATIONARY MODELS}

We consider an inflationary model with the inflaton potential of the form $V=(\lambda / 4) \phi^{4}$ during reheating. ${ }^{2}$ The inflaton field $\phi$ starts oscillating when the inflationary epoch ends at a cosmic time $t=t_{\mathrm{osc}}$. While oscillating the field $\phi$ decays and the decay products thermalise, ${ }^{3}$ and thus reheating occurs. ${ }^{4}$ Assuming that the universe

1 Refs. 25, 26] indicate regions of parameter space for a quartic potential for which bosonic and fermionic preheating can be suppressed. Furthermore, certain parameter values for which preheating is strong produce large non-gaussianities in the CMBR and are hence ruled out by WMAP [27].

${ }^{2}$ A quartic term can generate a quadratic term as well. We presume that this term, or any other mass term, is small and does not dominate till $\phi$ has almost decayed away.

3 By decay we refer to dissipation of the energy density of the inflaton field due to its coupling with other species.

${ }^{4}$ We assume that the inflaton products thermalise quickly as discussed in Appendix A of Ref. [13]. Refs. [36, 37] discuss an alternate description of reheating in the context of the MSSM. They argue that in the presence of large vevs for flat directions of MSSM fields thermalisation slows down. However if the vevs are is reheated through the perturbative decay of the inflaton field, the reheating picture, in general, can be described by [12]

$$
\begin{aligned}
\dot{\rho}_{r}+4 H \rho_{r} & =\Gamma_{\phi} \rho_{\phi} \\
H^{2} & =\frac{8 \pi G}{3}\left(\rho_{r}+\rho_{\phi}\right),
\end{aligned}
$$

where $\rho_{r}$ and $\rho_{\phi}$ are the energy densities of radiation and the inflaton respectively and $\Gamma_{\phi}$ is the rate of dissipation of the inflaton field energy density. Since the equation of state for the oscillating inflaton field and for radiation in Eq. (2) is the same we can write the rhs of Eq. (2) as $H_{I}^{2}\left(R_{\mathrm{osc}} / R\right)^{4}$, where $H_{I}$ is the Hubble parameter at $t_{\mathrm{osc}}$.

$$
H_{\mathrm{I}}=\sqrt{\frac{8 \pi}{3}} \frac{M_{I}^{2}}{M_{\mathrm{Pl}}},
$$

where $M_{I}=V_{I}^{1 / 4}, V_{I}$ being the inflaton energy density at $t_{\mathrm{osc}}$. Solving Eq.(2) then gives

$$
R=R_{\mathrm{osc}}\left[2 H_{\mathrm{I}}\left(t-t_{\mathrm{osc}}\right)+1\right]^{1 / 2} .
$$

(For $t \gg t_{\mathrm{osc}}, R \sim t^{\frac{1}{2}}$.)

Taking $\rho_{\phi}=M_{I}^{4}\left(R_{\mathrm{osc}} / R\right)^{4} \exp \left[-\Gamma_{\phi}\left(t-t_{\mathrm{osc}}\right)\right]$ in Eq. (11) we then get

$$
\rho_{r}=\frac{3}{8 \pi} M_{\mathrm{Pl}}^{2} H_{\mathrm{I}}^{2} \frac{1-\mathrm{e}^{-\Gamma_{\phi}\left(\mathrm{t}-\mathrm{t}_{\mathrm{osc}}\right)}}{\left[2 H_{\mathrm{I}}\left(t-t_{\mathrm{osc}}\right)+1\right]^{2}} .
$$

However to simplify our subsequent analysis, we ignore the change in $\rho_{\phi}$ due to decay in Eq. (11), which is valid till $t \lesssim t_{\text {reh }} \approx \Gamma_{\phi}^{-1} .{ }^{5}$ Then $\rho_{\phi} \approx M_{I}^{4}\left(R_{\mathrm{osc}} / R\right)^{4}$ and the solution of Eq. (11) is given by

$$
\begin{aligned}
\rho_{r} & =\frac{3}{8 \pi} M_{\mathrm{Pl}}^{2} \Gamma_{\phi} H_{\mathrm{I}}^{2} \frac{t-t_{\mathrm{osc}}}{\left[2 H_{\mathrm{I}}\left(t-t_{\mathrm{osc}}\right)+1\right]^{2}} \\
& =\sqrt{\frac{3}{32 \pi}} M_{I}^{2} \Gamma_{\phi} M_{\mathrm{Pl}}\left(\frac{R}{R_{\mathrm{osc}}}\right)^{-2}\left[1-\left(\frac{R}{R_{\mathrm{osc}}}\right)^{-2}\right](7)
\end{aligned}
$$

From Eq. (17) we see that during reheating the energy density initially increases to a maximum value

$$
\rho_{r}^{\max }=\frac{1}{4} \sqrt{\frac{3}{32 \pi}} \Gamma_{\phi} M_{\mathrm{Pl}} M_{I}^{2}
$$

at $R_{\max }=\sqrt{2} R_{\mathrm{osc}}$. The maximum temperature during reheating is then

$$
T_{\max }=0.6 g_{*}^{-1 / 4}\left(\Gamma_{\phi} M_{\mathrm{Pl}}\right)^{1 / 4} M_{I}^{1 / 2} .
$$

small $\left(<10^{-6} M_{\mathrm{Pl}}\right)$ then there is no effect on reheating. Furthermore the condensates of MSSM fields can fragment into Q-balls and the vevs can vanish in large parts of the universe [38]. These are the cases we would be considering.

5 We will assume for now that our analysis below is valid till $t_{\text {reh }}$ and will later discuss this assumption. 
Subsequently the temperature falls as $1 / R^{\frac{1}{2}}$ (for $t \gg$ $\left.t_{\max }\right)$ until the inflaton decays at $t_{\text {reh }}$. Once the final decay products of $\phi$ thermalise with each other through sufficient interactions the radiation density becomes

$$
\rho_{r}^{\mathrm{reh}}=\frac{\pi^{2}}{30} g_{*} T_{\mathrm{reh}}^{4} .
$$

From equations (6) and (10), and assuming $t_{\mathrm{reh}} \gg t_{\mathrm{osc}}$, we get the reheating temperature

$$
T_{\mathrm{reh}} \approx 0.55 g_{*}^{-1 / 4}\left(M_{\mathrm{Pl}} \Gamma_{\phi}\right)^{1 / 2} .
$$

In the following we examine the production of gravitinos during reheating, i.e., from $t_{\mathrm{osc}}$ to $t_{\mathrm{reh}}$, and during the subsequent radiation dominated era after $t_{\text {reh }}$, and discuss its consequences.

\section{GRAVITINO PRODUCTION}

Gravitinos are produced by the scattering of the inflaton decay products; a list of processes is provided in, for example, Tables 1 in Refs. [5, 10]. The Boltzmann equation for gravitinos is given by

$$
\frac{d n_{\tilde{G}}}{d t}+3 H n_{\tilde{G}}=\left\langle\Sigma_{\mathrm{tot}}|v|\right\rangle n^{2},
$$

where $n=\left(\zeta(3) / \pi^{2}\right) T^{3}$ is the number density of scatterers $(\zeta(3)=1.20206$.. is the Riemann zeta function of 3$), \Sigma_{\text {tot }}$ is the total scattering cross section for gravitino production, $v$ is the relative velocity of the incoming particles, and $\langle\ldots\rangle$ refers to thermal averaging. Since the gravitino lifetime is $10^{7-8}\left(100 \mathrm{GeV} / m_{\tilde{G}}\right) \mathrm{s}$ [5] decays are not relevant during the epoch of gravitino production for gravitinos of mass $10^{2-3} \mathrm{GeV}$. Hence we have not included the gravitino decay term in Eq. (12). We may now re-express this equation as

$$
\dot{T} \frac{d n_{\tilde{G}}}{d T}+3 H n_{\tilde{G}}=\left\langle\Sigma_{\text {tot }}|v|\right\rangle n^{2},
$$

(keeping in mind that $\dot{T}$ passes through zero at $T_{\max }$ ).

The cross section $\left\langle\Sigma_{\text {tot }}|v|\right\rangle$ is given by [39]

$$
\begin{aligned}
\left\langle\Sigma_{\mathrm{tot}}|v|\right\rangle \equiv \frac{\alpha}{M^{2}} & \\
=\frac{1}{M^{2}} \frac{3 \pi}{16 \zeta(3)} \sum_{i=1}^{3} & {\left[1+\frac{M_{i}^{2}}{3 m_{\tilde{G}}^{2}}\right] } \\
& \times c_{i} g_{i}^{2} \ln \left(\frac{k_{i}}{g_{i}}\right)
\end{aligned}
$$

where $i=1,2,3$ refers to the three gauge groups $U(1)_{Y}, S U(2)_{L}$ and $S U(3)_{c}$ respectively. $\quad M=$ $M_{\mathrm{Pl}} / \sqrt{8 \pi} \simeq 2.4 \times 10^{18} \mathrm{GeV}$ is the reduced Planck mass. $M_{i}$ are the gaugino masses. $g_{i}(T)$ are the gauge coupling constants, while $c_{i}$ and $k_{i}$ are constants associated with the gauge groups. $c_{1,2,3}$ are 11,27 and 72 and $k_{1,2,3}$ are 1.266, 1.312, 1.271 respectively (Table 1 of Ref. 39]). The above expression includes corrections to earlier expressions for the cross section for gravitino production in Refs. [40] and [17]. Using the one loop $\beta$-function of MSSM, the solution of the renormalization group equation for the gauge coupling constants is given by

$$
g_{i}(T) \simeq\left[g_{i}^{-2}\left(M_{Z}\right)-\frac{b_{i}}{8 \pi^{2}} \ln \left(T / M_{Z}\right)\right]^{-1 / 2},
$$

with $b_{1}=11, b_{2}=1, b_{3}=-3$. To obtain a conservative estimate of the gravitino abundance we take $M_{i} \rightarrow 0$ as in Ref. [17.

\section{Gravitino production during reheating}

Since $\dot{T}$ is zero at $T_{\max }$ we solve Eq. (13) from $t_{\mathrm{osc}}$ to $t_{\max }$ and from $t_{\max }$ to $t_{\mathrm{reh}}$ separately. In order to solve Eq. (13) we need $\dot{T}$ and $H$ as functions of $T$. Eq. (7) implies that

$$
R^{4} T^{4}-A R_{\mathrm{osc}}^{2} R^{2}+A R_{\mathrm{osc}}^{4}=0
$$

where with some algebra one can show that $A=4 T_{\max }^{4}$. Then in the epoch $t_{\mathrm{osc}} \leq t \leq t_{\max }$ we have

$$
R^{2} / R_{\mathrm{osc}}^{2}=\frac{1-\left(1-4 T^{4} / A\right)^{\frac{1}{2}}}{2 T^{4} / A}
$$

while for $t_{\max } \leq t \leq t_{\text {reh }}$

$$
R^{2} / R_{\mathrm{osc}}^{2}=\frac{1+\left(1-4 T^{4} / A\right)^{\frac{1}{2}}}{2 T^{4} / A} .
$$

Eq. (18) implies that for $t \gg t_{\max }, T \sim R^{-\frac{1}{2}}\left(\right.$ not $\sim R^{-1}$ as inflaton decay is a source of radiation). We now define a dimensionless variable

$$
x^{2}=1-\frac{4 T^{4}}{A}=1-\frac{T^{4}}{T_{\max }^{4}},
$$

Then the Hubble expansion parameter $H=H_{I}\left(R_{\mathrm{osc}} / R\right)^{2}$ can be rewritten as a function of $T$ or $x$ as

$$
H=\frac{1}{2} H_{I}(1+x) \quad \text { for } \quad t_{\mathrm{osc}} \leq t \leq t_{\max }
$$

and

$$
H=\frac{1}{2} H_{I}(1-x) \quad \text { for } \quad t_{\max } \leq t \leq t_{\mathrm{reh}} .
$$

For $\dot{T}$ we differentiate $\rho_{r}(T)=\left(\pi^{2} / 30\right) g_{*} T^{4}$ and $\rho_{r}(R)$ in Eq. (7) with respect to time, equate the results and get

$$
\dot{T}=\frac{30}{\pi^{2} g_{*}} \frac{1}{4 T^{3}} \frac{d \rho_{r}}{d R} \dot{R}=\frac{T}{4 \rho_{r}} \frac{d \rho_{r}}{d R} R H .
$$


Now using Eqs. (7), (17/18), (19) and (20|21) the above equation can be recast as

$$
\dot{T}=\frac{T_{\max }^{4} H_{I}}{2 T^{3}} x(1+x)^{2} \quad \text { for } \quad t_{\mathrm{osc}} \leq t \leq t_{\max }
$$

and

$$
\dot{T}=\frac{T_{\max }^{4} H_{I}}{2 T^{3}}(-x)(1-x)^{2} \quad \text { for } \quad t_{\max } \leq t \leq t_{\mathrm{reh}} .
$$

We now solve the Boltzmann equation Eq. (13) in the two regimes $t_{\mathrm{osc}} \leq t \leq t_{\max }$ and $t_{\max } \leq t \leq t_{\mathrm{reh}}$.

\section{Epoch: $t_{\mathrm{osc}} \leq t \leq t_{\max }$}

Using Eqs. (20) and (23) Eq. (13) can be written as

$$
\frac{d n_{\tilde{G}}}{d x}-\frac{d_{1}}{(1+x)} n_{\tilde{G}}=d_{2} \frac{\left(1-x^{2}\right)^{3 / 2}}{(1+x)^{2}}
$$

where

$$
d_{1}=\frac{3}{2} \text { and } d_{2}=-\frac{\alpha}{M^{2}}\left(\frac{\zeta(3)}{\pi^{2}}\right)^{2}\left(\frac{T_{\max }^{6}}{H_{I}}\right) .
$$

Now we define $y=1+x$, so that Eq. (25) can be rewritten as

$$
\frac{d n_{\tilde{G}}}{d y}-\frac{d_{1}}{y} n_{\tilde{G}}=d_{2} \frac{(2-y)^{3 / 2}}{y^{1 / 2}}
$$

Solving Eq. (27) from $y_{\text {osc }}=2$ to $y$ and assuming that $n_{\tilde{G}}\left(y_{\text {osc }}\right)=0$ we get the gravitino abundance as [4]

$$
\begin{aligned}
n_{\tilde{G}}(y)= & d_{2} y^{3 / 2}\left[\frac{2(2-y)-6}{y} \sqrt{2-y}\right. \\
& \left.-\frac{3}{\sqrt{2}} \ln \left|\frac{\sqrt{2-y}-\sqrt{2}}{\sqrt{2-y}+\sqrt{2}}\right|\right] .
\end{aligned}
$$

Thus at $y=y_{\max }=1$, which corresponds to $T=T_{\max }$, we get the gravitino abundance to be

$$
\begin{aligned}
n_{\tilde{G}}\left(y_{\max }\right) & =d_{2}\left[-4-\frac{3}{\sqrt{2}} \ln \left|\frac{1-\sqrt{2}}{1+\sqrt{2}}\right|\right] \\
& =-0.26 d_{2}
\end{aligned}
$$

Epoch: $t_{\max } \leq t \leq t_{\text {reh }}$

Using Eqs. (21) and (24) Eq. (13) can be written as

$$
\frac{d n_{\tilde{G}}}{d x}+\frac{d_{1}}{(1-x)} n_{\tilde{G}}=d_{2} \frac{\left(1-x^{2}\right)^{3 / 2}}{(1-x)^{2}}
$$

where $d_{1}$ and $d_{2}$ are given by Eq. (26). Defining $y=1-x$ Eq. (30) can be rewritten as

$$
\frac{d n_{\tilde{G}}}{d y}-\frac{d_{1}}{y} n_{\tilde{G}}=d_{2} \frac{(2-y)^{3 / 2}}{y^{1 / 2}}
$$

Solving Eq. (31) from $y_{\max }$ to $y$ we get the gravitino abundance as [41]

$$
\begin{aligned}
n_{\tilde{G}}(y)= & n_{\tilde{G}}\left(y_{\max }\right)\left(\frac{y}{y_{\max }}\right)^{3 / 2} \\
+ & d_{2} y^{3 / 2}\left[4+\frac{3}{\sqrt{2}} \ln \left|\frac{1-\sqrt{2}}{1+\sqrt{2}}\right|\right] \\
+ & d_{2} y^{3 / 2}\left[-2\left(1+\frac{1}{y}\right) \sqrt{2-y}\right. \\
& \left.-\frac{3}{\sqrt{2}} \ln \left|\frac{\sqrt{2-y}-\sqrt{2}}{\sqrt{2-y}+\sqrt{2}}\right|\right]
\end{aligned}
$$

Now using Eq. (29) and letting $y_{\max }=1$ and $y=y_{\text {reh }}$ the above equation can be written as

$$
\begin{aligned}
n_{\tilde{G}}\left(y_{\mathrm{reh}}\right)= & d_{2} y_{\mathrm{reh}}^{3 / 2}\left[-2\left(1+\frac{1}{y_{\mathrm{reh}}}\right) \sqrt{2-y_{\mathrm{reh}}}\right. \\
& \left.-\frac{3}{\sqrt{2}} \ln \left|\frac{\sqrt{2-y_{\mathrm{reh}}}-\sqrt{2}}{\sqrt{2-y_{\mathrm{reh}}}+\sqrt{2}}\right|\right]
\end{aligned}
$$

Using $y_{\text {reh }} \approx(1 / 2)\left(T_{\text {reh }} / T_{\max }\right)^{4}$ for $T_{\text {reh }} \ll T_{\max }$ we get the gravitino abundance at $T_{\text {reh }}$ as

$$
\begin{aligned}
n_{\tilde{G}}\left(T_{\mathrm{reh}}\right)= & \frac{d_{2}}{2^{3 / 2}}\left(T_{\mathrm{reh}} / T_{\max }\right)^{6} \\
& {\left[-4 \sqrt{2}\left(\frac{T_{\max }}{T_{\mathrm{reh}}}\right)^{4}\left(1+\frac{3}{8} \frac{T_{\mathrm{reh}}^{4}}{T_{\max }^{4}}-\frac{1}{16} \frac{T_{\mathrm{reh}}^{8}}{T_{\max }^{8}}\right)\right.} \\
- & \left.\frac{3}{\sqrt{2}} \ln \left(\frac{8 T_{\mathrm{reh}}^{4}}{16 T_{\max }^{4}-T_{\mathrm{reh}}^{4}}\right)\right]
\end{aligned}
$$

For $T_{\text {reh }} \ll T_{\max }$ the dominant contribution comes from the $\left(T_{\max } / T_{\text {reh }}\right)^{4}$ term in the square brackets. Then we can approximate the gravitational abundance at $T_{\text {reh }}$ as

$$
\begin{aligned}
n_{\tilde{G}}\left(T_{\mathrm{reh}}\right) & \simeq \frac{d_{2}}{2^{3 / 2}}\left(T_{\mathrm{reh}} / T_{\max }\right)^{6}\left[-4 \sqrt{2}\left(\frac{T_{\mathrm{max}}}{T_{\mathrm{reh}}}\right)^{4}\right] \\
& =2\left(\frac{\alpha}{M^{2}}\right)\left(\frac{\zeta(3)}{\pi^{2}}\right)^{2} \frac{T_{\max }^{4}}{H_{I}} T_{\mathrm{reh}}^{2} .
\end{aligned}
$$

\section{Gravitino production in the radiation dominated era}

After the inflaton field decays at $t_{\text {reh }}$ the universe enters the radiation dominated era. Unlike the reheating era during which the entropy continuously increases, in the radiation dominated era the total entropy remain constant (except for epochs of out-of-equilibrium decays). Therefore it is useful to express the abundance of any species $i$ as $Y_{i}=n_{i} / s$, where $n_{i}$ is the number density of the species $i$ in a physical volume and $s$ is the entropy density given by

$$
s=\frac{2 \pi^{2}}{45} g_{*} T^{3}
$$




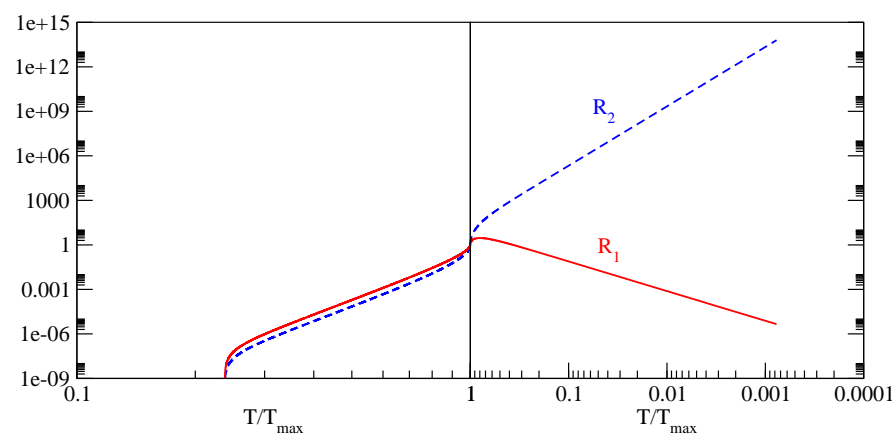

FIG. 1: The production of gravitinos is shown from $t_{\mathrm{osc}}$ to $t_{\text {reh }}$ through $t_{\max }$ as a function of temperature for values mentioned in the Discussion. The temperature rises from 0 at $t_{\mathrm{osc}}$ to $T_{\max }$ at $t_{\max }$ and then falls to $T_{\text {reh }}$ at $t_{\text {reh. }}$. The (normalised) gravitino number density $\mathrm{R}_{1}=n_{\tilde{G}}(T) / n_{\tilde{G}}\left(T_{\max }\right)$ rises from 0 to its maximum value at $t_{\mathrm{MAX}}>t_{\max }$, when $T_{\mathrm{MAX}} \simeq 0.8 T_{\max }$, and then decreases till $t_{\mathrm{reh}}$ [red solid line]. The (normalised) gravitino number density per comoving volume $\mathrm{R}_{2}=\bar{n}_{G}(T) / \bar{n}_{G}\left(T_{\max }\right)$ is also plotted [blue dashed line].

We take $g_{*}=228.75$ in the MSSM for the temperature range of interest. One can now re-express Eq. (13) as

$$
\dot{T} \frac{d Y_{\tilde{G}}}{d T}=\left\langle\Sigma_{\text {tot }}|v|\right\rangle Y n
$$

To obtain $\dot{T}$ we use the temperature-time relation for the radiation dominated era, namely,

$$
T=T_{\mathrm{reh}} \frac{1}{\left[2 H_{\mathrm{reh}}\left(t-t_{\mathrm{reh}}\right)+1\right]^{\frac{1}{2}}},
$$

where

$$
H_{\mathrm{reh}}=\sqrt{\frac{8 \pi^{3} g_{* \mathrm{reh}}}{90}} \frac{T_{\mathrm{reh}}^{2}}{M_{\mathrm{Pl}}} .
$$

(For $t \gg t_{\text {reh }}, T \sim t^{-\frac{1}{2}}$.) Therefore $\dot{T}$ is given by

$$
\dot{T}=-\frac{H_{\text {reh }}}{T_{\text {reh }}^{2}} T^{3}=-\left(\frac{g_{* \mathrm{reh}} \pi^{2}}{90}\right)^{\frac{1}{2}} \frac{T^{3}}{M} .
$$

Then

$$
\frac{d Y_{\tilde{G}}}{d T}=-\left(\frac{90}{g_{* \mathrm{reh}} \pi^{2}}\right)^{1 / 2}\left(\frac{45}{2 \pi^{2} g_{*}}\right)\left(\frac{\alpha}{M}\right)\left(\frac{\zeta(3)}{\pi^{2}}\right)^{2} .
$$

Assuming $\alpha$ to be independent of temperature and integrating the above equation from $T_{\text {reh }}$ to $T_{\mathrm{f}}$, the final temperature, we get the total gravitino abundance at $T_{\mathrm{f}}$ to be

$$
Y_{\tilde{G}}\left(T_{\mathrm{f}}\right)=Y_{\tilde{G}}\left(T_{\mathrm{reh}}\right)+Y_{\tilde{G}}^{\mathrm{rad}}\left(T_{f}\right)
$$

where

$$
\begin{aligned}
Y_{\tilde{G}}^{\mathrm{rad}}\left(T_{f}\right)= & \left(\frac{90}{g_{* \mathrm{reh}} \pi^{2}}\right)^{1 / 2}\left(\frac{45}{2 \pi^{2} g_{* \mathrm{reh}}}\right) \\
& \times\left(\frac{\alpha}{M}\right)\left(\frac{\zeta(3)}{\pi^{2}}\right)^{2}\left(T_{\mathrm{reh}}-T_{\mathrm{f}}\right)
\end{aligned}
$$

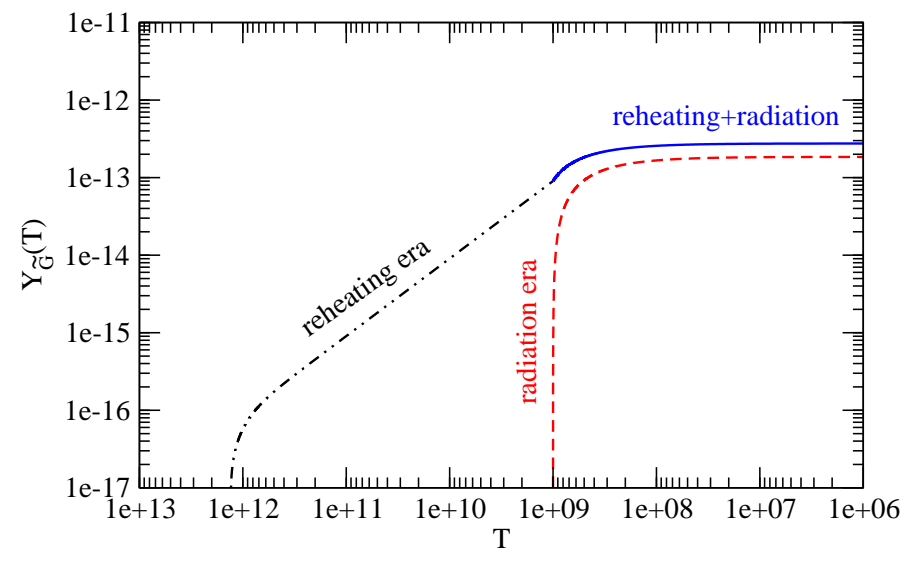

FIG. 2: $\quad Y_{\tilde{G}}=n_{\tilde{G}} / s$ generated during the reheating era, the radiation dominated era, and the sum of contributions from both eras are shown as a function of the temperature $T$ for $t>t_{\max } . T_{\text {reh }}$ and $M_{I}$ are chosen to be $10^{9} \mathrm{GeV}$ and $10^{16} \mathrm{GeV}$ respectively, and so $T_{\max } \approx 1.3 \times 10^{12} \mathrm{GeV}$. Since $Y_{\tilde{G}}$ in both eras is largely generated close to $T_{\text {reh }}, \alpha$ is evaluated at $T_{\text {reh }}$. The final value of $Y_{\tilde{G}}$ is $\approx 3 \times 10^{-13}$.

is the gravitino abundance produced in the radiation dominated era. We have used $g_{* \text { reh }}$ in the expression for $Y_{\tilde{G}}^{\mathrm{rad}}$ and ignored the variation of $g_{*}$ with temperature. This is justified since most of the gravitinos are generated close to $T_{\text {reh }}$. Using Eqs. (35) and (36)

$$
Y_{\tilde{G}}\left(T_{\mathrm{reh}}\right)=\frac{2 \alpha}{M^{2}}\left(\frac{\zeta(3)}{\pi^{2}}\right)^{2}\left(\frac{45}{2 \pi^{2} g_{* \mathrm{reh}}}\right) \frac{T_{\max }^{4}}{H_{I} T_{\mathrm{reh}}} .
$$

This contribution is usually neglected while obtaining the gravitino bound. As we see below this is comparable with the second term in Eq. (42). Using Eqs. (43) and (44) in Eq. (42) we get

$$
\begin{aligned}
Y_{\tilde{G}}\left(T_{\mathrm{f}}\right)= & \frac{\alpha}{M^{2}}\left(\frac{\zeta(3)}{\pi^{2}}\right)^{2}\left(\frac{45}{2 \pi^{2} g_{* \mathrm{reh}}}\right)\left[2 \frac{T_{\max }^{4}}{H_{I} T_{\mathrm{reh}}}\right. \\
& \left.+M\left(\frac{90}{g_{* \mathrm{reh}} \pi^{2}}\right)^{1 / 2} T_{\mathrm{reh}}\right]
\end{aligned}
$$

where we have used $T_{\mathrm{f}} \ll T_{\text {reh }}$. Relating $T_{\max }$ to $T_{\text {reh }}$ from Eqs. (3), (9) and (11) the total gravitino abundance is then given by

$$
\begin{aligned}
Y_{\tilde{G}}\left(T_{f}\right)= & \frac{3 \alpha T_{\mathrm{reh}}}{M}\left(\frac{\zeta(3)}{\pi^{2}}\right)^{2}\left(\frac{45}{2 \pi^{2} g_{* \mathrm{reh}}^{3 / 2}}\right) \\
& {[0.49+1.0], }
\end{aligned}
$$

where we have used $g_{* \text { reh }}$ in the expressions for $T_{\max }$.

\section{DISCUSSION}

The detailed dynamics of gravitino production from $t_{\mathrm{osc}}$ to $t_{\mathrm{reh}}$ is shown in Fig. (11) as a function of the 
temperature using Eqs. (28) and (32). We normalise the gravitino number density with respect to the value at $T_{\max }$. $T_{\text {reh }}$ and $M_{I}$ are chosen to be $10^{9} \mathrm{GeV}$ and $10^{16} \mathrm{GeV}$ respectively, and so $T_{\max } \approx 1.3 \times 10^{12} \mathrm{GeV} . \alpha$ is treated as constant and evaluated at $T_{\text {reh }}$ since most gravitinos are produced near $T_{\text {reh }} . \alpha$ is 15.1 , using $g_{i}\left(M_{z}\right)$ obtained from $\alpha_{E M}\left(M_{Z}\right)=1 / 128, \sin ^{2} \theta_{W}\left(M_{Z}\right)=0.231$, $\alpha_{s}\left(M_{Z}\right)=0.119$, and $M_{Z}=91.2 \mathrm{GeV}$ [42].

It can be seen in Fig. (11) that during reheating the number density of gravitinos monotonically increases from $t_{\mathrm{osc}}$ to a time $t_{\mathrm{MAX}}>t_{\mathrm{max}}$, where $t_{\mathrm{MAX}}$ corresponds to a temperature $T_{\mathrm{MAX}} \simeq 0.8 T_{\max }$ and $n_{\tilde{G}}\left(t_{\mathrm{MAX}}\right) \approx$ $3 n_{\tilde{G}}\left(t_{\max }\right)$. Subsequently it decreases till $t_{\text {reh }}$ to a value $7.7\left(T_{\mathrm{reh}} / T_{\max }\right)^{2} n_{\tilde{G}}\left(t_{\max }\right)$. Taking the number density per comoving volume as $\bar{n}_{G}(T)=\left(n_{\tilde{G}} R^{3}\right) / R_{\text {osc }}^{3}$ we also plot $\bar{n}_{G}$ (normalised to the value at $T_{\max }$ ) by using Eqs. (17), (18), (28) and (32). From the plot of $\bar{n}_{G}(T)$ in Fig. (11) it can be seen that most gravitinos during reheating are produced close to $T_{\text {reh }}$.

In Fig. (2) we show the contribution to the gravitino abundance from the reheating era and from the subsequent radiation dominated era, and the sum of these contributions. From Eq. (46) it is clear that the gravitino production during the reheating era is almost half of that during the radiation dominated era even though a priori one would not have expected the gravitino production in both these eras to be similar. While the gravitino abundance generated during reheating is a function of $T_{\max }$ it is interesting that it can be re-expressed as independent of $T_{\max }$, and as a function of only $T_{\text {reh }}$. Moreover, the contribution to $Y_{\tilde{G}}$ from the reheating era is linearly proportional to $T_{\text {reh }}$, as it is for the radiation dominated era. These results are similar to those obtained in Ref. [16]. The linear dependence on $T_{\text {reh }}$ makes it simple to revise the constraints on $T_{\text {reh }}$ based on the upper limit on the gravitino abundance - the upper bound on $T_{\text {reh }}$ is lowered by a factor of $3 / 2$. Since $T_{\max } \propto \sqrt{T}_{\text {reh }}, T_{\max }$ is not affected much. Therefore models of leptogenesis that invoke a large $T_{\max }$ to create heavy Majorana neutrinos are not significantly impacted.

Above we partly ignored inflaton decay in our analysis, i.e., we did not include the effect of $\exp \left[-\Gamma_{\phi}\left(t-t_{\mathrm{osc}}\right)\right]$ in $\rho_{\phi}$ in Eq. (1). One might be concerned that this will lead to inaccuracies close to $t_{\text {reh }}$ when most of the gravitinos are produced. However if one writes $\rho_{\phi} \sim R^{-4} \exp \left(-\Gamma_{\phi} t\right) \sim t^{-2} \exp \left(-\Gamma_{\phi} t\right)$ for $t \gg t_{\max }$ then $\dot{\rho}_{\phi} / \rho_{\phi}=-2 / t-\Gamma_{\phi}$. Therefore even till close to $t_{\text {reh }}=\Gamma_{\phi}^{-1} \rho_{\phi}$ decreases primarily due to the expansion of the universe. Furthermore, if we follow the value of $Y_{\tilde{G}}=n_{\tilde{G}} / s$ we find (from Eq. (44)) that near $t_{\text {reh }}$ it increases as $T^{-1} \sim R^{\frac{1}{2}} \sim t^{\frac{1}{4}}$. At $t \approx 0.1 \Gamma_{\phi}^{-1} 56 \%$ of our estimate of $Y_{\tilde{G}}$ is already generated while decay has led to a reduction in $\rho_{\phi}$ of only $9 \%$. Keeping in mind the above arguments, we expect that the error in our estimate of $Y_{\tilde{G}}$ will not be large. A more accurate estimate will require a numerical analysis. (For the quadratic potential,
Ref. [16] (analytic) and Ref. [17] (numerical) obtained a gravitino abundance of $1.9 \times 10^{-13}$ and $1.5 \times 10^{-13}$ respectively for $T_{\text {reh }}$, as defined in Ref. [16], set to $10^{9} \mathrm{GeV}$. $\left.{ }^{6}\right)$

\section{CONCLUSION}

In conclusion, in this article we have calculated the gravitino abundance generated during reheating for an inflationary model with a quartic potential during reheating. We find that the gravitino abundance generated during reheating is a function of the largest temperature during reheating. However it can be re-expressed in terms of the reheat temperature only and we find that it is linearly proportional to the the reheat temperature, as in the standard calculation of gravitinos produced in the radiation dominated era after reheating. Furthermore, we find that this abundance is $49 \%$ of the abundance of gravitinos generated in the radiation dominated era. This lowers the upper bound on the reheat temperature by a factor of $3 / 2$. However this does not significant change the viability of leptogenesis scenarios.

Acknowledgement: NS would like to thank Anupam Mazumdar and Kazunori Kohri for useful discussions. NS was supported by the European Union through the Marie Curie Research and Training Network "UniverseNet" (MRTN-CT-2006-035863).

* Electronic address: raghavan@prl.res.in

$\dagger$ Electronic address: n.sahu@lancaster.ac.uk

[1] D. V. Nanopoulos, K. A. Olive and M. Srednicki, After Primordial Inflation, Phys. Lett. B 12730 (1983).

[2] L. M. Krauss, New Constraints On 'Ino' Masses From Cosmology. 1. Supersymmetric 'Inos', Nucl. Phys. B 227 556 (1983).

[3] I. V. Falomkin, G. B. Pontecorvo, M. G. Sapozhnikov, M. Yu. Khlopov, F. Balestra and G. Piragino, LowEnergy Anti-P He-4 Annihilation And Problems Of The Modern Cosmology, GUT And Susy Models, Nuovo Cim. A 79, 193 (1984) [Yad. Fiz. 39, 990 (1984)].

[4] M. Yu. Khlopov and A. D. Linde, Is It Easy To Save The Gravitino?, Phys. Lett. B 138265 (1984).

[5] J. Ellis, J. E. Kim and D. V. Nanopoulos, Cosmological Gravitino Regeneration And Decay, Phys. Lett. B 145 181 (1984).

[6] R. Juszkiewicz, J. Silk and A. Stebbins, Constraints On Cosmologically Regenerated Gravitinos, Phys. Lett. B158 463 (1985).

6 Refs. [16] and [17] define the reheat temperature differently. Note that both their results are enhanced by $27 \%$ if one uses the gravitino production rate from Ref. [39]. 
[7] J. Ellis, D. V. Nanopoulos and S. Sarkar, The Cosmology Of Decaying Gravitinos, Nucl. Phys. B 259175 (1985).

[8] M. Kawasaki and K. Sato, Decay Of Gravitinos And Photodestruction Of Light Elements, Phys. Lett. B189 23 (1987) .

[9] M. Yu. Khlopov, Yu. L. Levitan, E. V. Sedel'nikov, and I. M. Sobol, Nonequilibrium cosmological nucleosynthesis of light elements: Calculations by the Monte Carlo method, Phys. Atom. Nucl. 571393 (1994) [Yad. Fiz. 57, 1466 (1994)].

[10] M. Kawasaki and T. Moroi, Gravitino production in the inflationary universe and the effects on big bang nucleosynthesis, Prog. Theor. Phys. 93, 879 (1995) arXiv:hep-ph/9403364.

[11] R. H. Cyburt, J. R. Ellis, B. D. Fields and K. A. Olive, Updated nucleosynthesis constraints on unstable relic particles, Phys. Rev. D 67103521 (2003) arXiv:astro-ph/0211258.

[12] E.W. Kolb and M. Turner, The Early Universe, AddisonWesley, Frontiers in Physics Series, 69 (1990), pp. 278281 .

[13] D. J. H. Chung, E. W. Kolb and A. Riotto, Production of massive particles during reheating, Phys. Rev. D 60, 063504 (1999) arXiv:hep-ph/9809453.

[14] G. F. Giudice, E. W. Kolb and A. Riotto, Largest temperature of the radiation era and its cosmological implications, Phys. Rev. D 64, 023508 (2001) arXiv:hep-ph/0005123.

[15] D. Delepine and U. Sarkar, Gravitino constraints on models of neutrino masses and leptogenesis, Phys. Rev. D 60, 055005 (1999) arXiv:hep-ph/9811479.

[16] R. Rangarajan and N. Sahu, Gravitino production in an inflationary universe: A fresh look, Mod. Phys. Lett. A 23, 427 (2008) arXiv:hep-ph/0606228.

[17] M. Kawasaki, K. Kohri and T. Moroi, Big-bang nucleosynthesis and hadronic decay of long-lived massive particles, Phys. Rev. D 71, 083502 (2005) arXiv:astro-ph/0408426.

[18] G. F. Giudice, A. Riotto and I. Tkachev, Thermal and non-thermal production of gravitinos in the early universe, JHEP 9911, 036 (1999) arXiv:hep-ph/9911302.

[19] J. Pradler and F. D. Steffen, Constraints on the reheating temperature in gravitino dark matter scenarios, Phys. Lett. B 648, 224 (2007) arXiv:hep-ph/0612291.

[20] See pp. 279 and 300 in Ref. 12].

[21] A. D. Dolgov and D. P. Kirilova, Production of particles by a variable scalar field, Sov. J. Nucl. Phys. 51, 172 (1990) [Yad. Fiz. 51, 273 (1990)].

[22] J. H. Traschen and R. H. Brandenberger, Particle production during out-of-equilibrium phase transitions, Phys. Rev. D 42, 2491 (1990).

[23] L. Kofman, A. D. Linde and A. A. Starobinsky, Reheating after inflation, Phys. Rev. Lett. 73, 3195 (1994) arXiv:hep-th/9405187.

[24] Y. Shtanov, J. H. Traschen and R. H. Brandenberger, Universe reheating after inflation, Phys. Rev. D 51, 5438 (1995) arXiv:hep-ph/9407247.

[25] P. B. Greene, L. Kofman, A. D. Linde and A. A. Starobinsky, Structure of resonance in preheating after inflation, Phys. Rev. D 56, 6175 (1997) arXiv:hep-ph/9705347.

[26] P. B. Greene and L. Kofman, Preheating of fermions,
Phys. Lett. B 448, 6 (1999) arXiv:hep-ph/9807339.

[27] A. Jokinen and A. Mazumdar, Very Large Primordial Non-Gaussianity from multi-field: Application to Massless Preheating, JCAP 0604, 003 (2006) arXiv:astro-ph/0512368.

[28] A. L. Maroto and A. Mazumdar, Production of spin 3/2 particles from vacuum fluctuations, Phys. Rev. Lett. 84, 1655 (2000) arXiv:hep-ph/9904206.

[29] R. Kallosh, L. Kofman, A. D. Linde and A. Van Proeyen, Gravitino production after inflation, Phys. Rev. D 61, 103503 (2000) arXiv:hep-th/9907124.

[30] S. Tsujikawa, B. A. Bassett and F. Viniegra, Multifield fermionic preheating, JHEP 0008, 019 (2000) arXiv:hep-ph/0006354.

[31] H. P. Nilles, M. Peloso and L. Sorbo, Nonthermal production of gravitinos and inflatinos, Phys. Rev. Lett. 87, 051302 (2001) arXiv:hep-ph/0102264.

[32] H. P. Nilles, M. Peloso and L. Sorbo, Coupled fields in external background with application to nonthermal production of gravitinos, JHEP 0104, 004 (2001) arXiv:hep-th/0103202.

[33] H. P. Nilles, K. A. Olive and M. Peloso, The inflatino problem in supergravity inflationary models, Phys. Lett. B 522, 304 (2001) arXiv:hep-ph/0107212.

[34] P. B. Greene, K. Kadota and H. Murayama, Supergravity inflation free from harmful relics, Phys. Rev. D 68, 043502 (2003) arXiv:hep-ph/0208276.

[35] D. I. Podolsky, G. N. Felder, L. Kofman and M. Peloso, Equation of state and beginning of thermalization after preheating, Phys. Rev. D 73, 023501 (2006) arXiv:hep-ph/0507096.

[36] R. Allahverdi and A. Mazumdar, Quasithermal universe: From cosmology to colliders, arXiv:hep-ph/0505050.

[37] R. Allahverdi and A. Mazumdar, Supersymmetric thermalization and quasi-thermal universe: Consequences for gravitinos and leptogenesis, JCAP 0610, 008 (2006) arXiv:hep-ph/0512227.

[38] R. Allahverdi and A. Mazumdar, Reheating in supersymmetric high scale inflation, Phys. Rev. D 76, 103526 (2007) arXiv:hep-ph/0603244.

[39] J. Pradler and F. D. Steffen, Thermal Gravitino Production and Collider Tests of Leptogenesis, Phys. Rev. D 75, 023509 (2007) arXiv:hep-ph/0608344.

[40] M. Bolz, A. Brandenburg and W. Buchmuller, Thermal production of gravitinos, Nucl. Phys. B 606, 518 (2001) [Erratum-ibid. B 790, 336 (2008)], arXiv:hep-ph/0012052.

[41] The differential equation can be solved using an integrating factor, $\mu$. While obtaining the solution using $\mu$ it is convenient to set $2-y=z$ and to then use $\int z^{\frac{3}{2}} /(2-z)^{2} d z=(2 z-6)(z-2)^{-1} \sqrt{z}+(3 / \sqrt{2}) \ln \mid(\sqrt{z}-$ $\sqrt{2}) /(\sqrt{z}+\sqrt{2}) \mid$ (see G. Petit Bois, Tables of Indefinite Integrals, Dover Publications, p. 66). Mathematica provides this integral in terms of hypergeometric functions. The expressions in Eqs. (28) and (32) are written differently for subsequent manipulation of Eq. (32) for small $y$.

[42] S. Eidelman et al., Particle physics data group, Phys. Lett. B 592, 1 (2004) http://pdg.lbl.gov . 